\title{
Self-Organizing Flows in Social Networks
}

\author{
Nidhi Hegde \\ Bell Labs France Alcatel-Lucent \\ France \\ nidhi.hegde@alcatel-lucent.com \\ Laurent Massoulié* \\ Microsoft Research - Inria Joint Centre \\ France \\ laurent.massoulie@inria.fr \\ Laurent Viennot ${ }^{\dagger}$ \\ Inria - Paris Diderot University \\ France \\ laurent.viennot@inria.fr
}

\begin{abstract}
Social networks offer users new means of accessing information, essentially relying on "social filtering", i.e. propagation and filtering of information by social contacts. The sheer amount of data flowing in these networks, combined with the limited budget of attention of each user, makes it difficult to ensure that social filtering brings relevant content to interested users. Our motivation in this paper is to measure to what extent self-organization of a social network results in efficient social filtering.

To this end we introduce flow games, a simple abstraction that models network formation under selfish dynamics, featuring user-specific

\footnotetext{
*Part of this work was done while at Technicolor.

†Supported by the Inria project-team "Gang" in the "LIAFA" laboratory and by the "LINCS" laboratory.
} 
interests and budget of attention. In the context of homogeneous user interests, we show that selfish dynamics converge to a stable network structure (namely a pure Nash equilibrium) with close-to-optimal information dissemination. We show that, in contrast, for the more realistic case of heterogeneous interests, selfish dynamics may lead to information dissemination that can be arbitrarily inefficient, as captured by an unbounded "price of anarchy".

Nevertheless the situation differs when user interests exhibit a particular structure, captured by a metric space with low doubling dimension. In that case, natural autonomous dynamics converge to a stable configuration. Moreover, users obtain all the information of interest to them in the corresponding dissemination, provided their budget of attention is logarithmic in the size of their interest set.

\section{Introduction}

Information access has been revolutionized by the advent of social networks such as Facebook, Google+ and Twitter. These platforms have brought about the new paradigm of "social filtering", whereby one accesses information by "following" social contacts.

This is especially true for twitter-like microblogging social networks. In such networks the functions of filtering, editing and disseminating news are totally distributed, in contrast to traditional news channels. The efficiency of social filtering is critically affected by the network topology, as captured by the contact-follower relationships. Today's networks provide recommendations to users for potentially useful contacts to follow, but don't interfere any further with topology formation. In this sense, these networks self-organize, under the selfish decisions of individual users.

This begs the following question: when does such autonomous and selfish self-organizing topology lead to efficient information dissemination? The answer will in turn indicate under what circumstances self-organization is insufficient, and thus when additional mechanisms, such as incentive schemes, should be introduced.

Two parameters play a key role in this problem. On the one hand each user aims to maximize the coverage of the topics of his interest. On the other hand, a user pays with his attention: filtering interesting information from spam (i.e. information that does not fall in his topics of interest) incurs a cost. Users must therefore trade-off topic coverage against attention cost. 
As pointed out by Simon [27], as information becomes abundant, another resource becomes scarce: attention.

Furthermore, there is an interplay between participants in a social network where filtering by one user may benefit another, inducing complex dependencies in decisions on creating connections. To model this, we introduce a network formation game called flow game where some users produce news about specific topics and each user is interested in receiving all news about a set of topics specific to him. Each user is a selfish agent that can choose his incoming connections within a certain budget of attention in order to maximize the coverage of his set of topics of interest.

This model is of interest on its own, as it enriches the class of existing network formation games with a focus on flow dissemination under bounded connections. This model could also be of interest in the context of peer-topeer streaming and file sharing or publish/subscribe applications.

\section{$1.1 \quad$ Our results}

An important feature in our model is a user's budget of attention for the consumption of content. In previous work [16] the budget of attention was modelled as a limit on the rate with which a user consults a friend, with a different objective of minimizing delay in receiving all content. In the present work we are interested in a more fundamental question, of how efficient social networks are formed in the first place. We consider the model where users are interested in specific subsets of topics and their objective is to maximize the number of flows received corresponding to these topics. As such, we model the budget of attention as a constraint on the number of connections a user may create (rather than a rate of consultation). Our aim is to build a simple model capturing the complexity of the problem. This way of capturing the budget of attention amounts to assuming that each connection consumes the same amount of attention. We discuss in Section 6 how we can tweak our model to more finely model attention consumption.

We capture users' interests in topics through user-specific values for each topic and define the utility a user receives to be the sum of values of all received topics. Each user's objective in a flow game is then to choose connections so as to maximize his utility. We additionally assume that a user may produce news about one topic at most even if he redistributes other topics. This is coherent with an empirical study of twitter traces [6] where it is shown that ordinary users (as opposed to celebrities or newspapers) can 
gain influence by concentrating on a single topic.

Our main results relate to the stability and efficiency of the formation of information flows. We derive conditions where selfish dynamics converge to a pure Nash equilibrium. We then give approximation ratios bounding the quality of an equilibrium compared to an optimal solution. This is traditionally measured through the price of anarchy, the ratio of the global welfare (measured as the sum of user utilities) at an optimal solution compared to that at the worst equilibrium.

More precisely, we first consider homogeneous games where all users are interested in the same set of topics. We can then prove that selfish dynamics always converge to an equilibrium. Selfish dynamics comprise of any sequence of moves, where in each move a user is given the opportunity to selfishly rewire his connections to increase his utility. We show moreover that convergence occurs within a polynomial number of rounds where a round is a sequence of selfish moves including at least one move per user. We additionally show that the price of anarchy is bounded and approaches 1 as the budget of attention of users increases.

In the more general case where users interests are heterogeneous, selfish dynamics may not converge and price of anarchy may be unbounded. However, we observe that fast convergence towards efficient configurations can occur when users' interests are captured by a metric space with sufficient structure. Here, the interests of a user are modeled a point in this space such that nearby topics are of interest to the user. Sufficient structure typically arises when the metric space is a Euclidean space with low dimension. Our results are tailored to the more general case of metrics with low doubling dimension. Low dimension assumptions are classically used in information retrieval when data can be viewed as a matrix which is approximated with a low rank matrix. For example, a ranking technique for the web is proposed in [14] using a 16-dimensional space for representing topics of web pages. Closer to the context of our study, modeling people's opinions as points in a low Euclidean space is a classical approach in social sciences. Political spectrum for example is often modeled as a one dimensional space along a Left-Right axis. consist in introducing more dimensions. This concept can be formalized with single-peaked preference curves [5]. An online system for exchanging political views could be a concrete example where the technical conditions of our model are met. We believe that the same applies for the various domains of interest of a user, implying that our model remains valid more broadly if we can attribute several points of interest (one per domain) 
to each user. An extension of our model in that direction is proposed.

\subsection{Related work}

Information spread in networks has been studied extensively. Much of the past work study the properties of information diffusion on given networks with given sharing protocols. Our goal in this work is to study how networks form when users create connections with the objective of efficient content dissemination in a game-theoretical approach. This work thus follows the large amount of work in network formation games. However, to the best of our knowledge, the objective of efficient information dissemination under edge constraints and interest sets that we consider here is novel. We now discuss some work in those domains that are most relevant to this paper.

A lot of attention has been given to simple models of diffusion in social networks such as "rumor spreading" or "cascading" where a piece of information interests all users and is propagated in the network through random interactions typically (see e.g. the related work mentioned in [12] for rumor spreading and [18] for cascading). In this paper, we are more interested in the selective propagation of information according to connections chosen locally for optimizing the coverage of personal interests.

Network formation games have been considered in previous work in economics and in the context of the formation of Internet peering relations and peer-to-peer overlay networks. Economic models of network formation [15] use edges to represent social relations and it is typically assumed that the creation of an edge needs bilateral agreement since both users benefit from an edge. Our model is oriented and unilateral agreement is more relevant to the notion of following in social networks. A non-cooperative one-way link connection game has been considered in previous work [3], where each created link incurs a cost and users are interested in connecting to all other users. Our model is richer and more realistic where we consider connections to subsets of information flows that hold user-specific intrinsic values.

Network creation games in the context of the Internet have been considered [23], where distributed formation of undirected edges with a linear cost on each edge formed is studied. In such games, each user's objective is to minimize total formation cost while either minimizing distance to all other users [8], or ensuring connection to a given subset of nodes [2]. We consider a bound on edge costs, in the form of a limit on the number of in-edges at each node, and further, we focus on connections that allow specific flows of 


\section{information.}

Interestingly, bounded budget network formation games have already been considered. Bounded budget connection games [19] consider a bound on each user's budget in creating edges, with the objective being the minimization of the sum of weighted distances to other nodes. A similar model is considered in [4] where each user's objective is to maximize his influence, measured using betweenness centrality. In our work however, rather than minimizing distance to any node, we consider a formation game with the objective of ensuring connections to a subset of flows of interest, without regard to the particular nodes.

The notion of connecting to users that can provide a content flow of interest is similar to peer-to-peer live streaming systems [20]. Unlike peerto-peer streaming, we do not aim to satisfy flow rates, rather our aim is to connect to as many sets of relevant flows as possible. Moreover, our model allows differing user interests. The stability of connecting users of a peerto-peer network according to some affinities between users was studied using b-matching and acyclic preference systems [11]. As a generalization of the stable marriages problem, those systems consider configurations of undirected edges based on mutual acceptance of an edge, whereas unilateral decision is more suitable in our model. Our model is more intricate in the sense that connections are based not only on preferences but also on complementarity of content obtained through various connections.

Most notably, a model similar to ours has been independently developed in [21]. The authors propose a model for explaining how social media can provide efficient filtering of information. They model online exchange of media information with three type of actors: official media sites that provide fresh news every day, bloggers that relay some of these news and users that access these news through bloggers. The model also includes a game-theoretical part where the players are the bloggers. The strategy of a blogger is the set of news he decides to relay and his utility is the number of users following him. Conversely to our model, this is not a network formation game. However, it could be interesting to see if both approaches can be mixed together for modeling multi-hop relaying where bloggers can also relay other bloggers.

In Section 5 we model the space of user interests by a metric space with low doubling dimension. Modeling interests of users through a metric space seems a natural approach and bounded growth metrics, or more generally doubling metrics, have shown to be very a general model [24] that can capture general situations, while still providing an algorithmic perspective. The 
doubling dimension extends the notion of dimension from Euclidean spaces to arbitrary metric spaces. It has proven to be useful in many application domains such as nearest neighbor queries to databases [7], network construction [1], closest server selction [17], etc. Doubling metrics have notably been used to model distances in networks such as Internet [10].

\subsection{Organization of the paper}

Section 2 introduces the model. We study the case of homogeneous interests in Section 3. The heterogeneous case in its full generality is considered in Section 4 which details some negative results. Section 5 is dedicated to the specific scenario where users' interests are captured by a doubling metric, enabling some positive results. Section 6 presents how the costs of attention can be better modeled with respect to the intersection of user interests. We finally conclude in Section 7 describing potential extensions of the current work.

\section{Model}

We consider a social network where users interested in some set of content topics (or subjects) connect to (or follow in social networking parlance) other users in order to obtain such contents, materialized by flows of news. Each user may produce news for at most one topic (but may forward news from other topics she is interested in). To distinguish the role of publisher from that of follower, we technically assume that news concerning a given topic (or subject) are produced at a given node called producer which is identified with that topic.

A flow game is defined as a tuple $(V, P, S, \Delta)$ where $V$ is a set of users, $P$ a set of producers (or subjects or topics) and $S: V \rightarrow P$ is a function associating to each user $u$ its interest set $S_{u} \subseteq P$, and $\Delta: V \rightarrow \mathbb{N}$ is a function associating to each user $u$ its budget of attention $\Delta_{u}$. We let $n=|V|$ and $p=|P|$ denote the number of users and producers respectively. A flow game is homogeneous if all users have the same interest set: $S_{u}=P$ for all $u \in V$. If this is not the case, the game is said to be heterogeneous.

A strategy for user $u$ is a subset $F_{u}$ of $\{(v, u): v \in V \cup P\}$ such that $\left|F_{u}\right| \leq \Delta_{u}\left(\Delta_{u}\right.$ is an upper bound on the in-degree of $u$ that we call the budget of $u$ ). For all $(v, u) \in F_{u}$, we say that $u$ follows $v$ or equivalently 
that $u$ is connected to $v$ (such a link $(v, u)$ created by $u$ is oriented according to the data flow, that is from $v$ to $u$ ). The collection $F=\left\{F_{u}: u \in V\right\}$ forms a network defined by the directed graph $G(F)=(V \cup P, E(F))$ where $E(F)=\cup_{u \in V} F_{u}$. A user $u$ is interested in a subject $s$ if $s \in S_{u}$. A user $u$ receives a subject $s \in P$ if there exists a directed path from $s$ to $u$ in $G(F)$ such that all intermediate nodes are interested in $s$. We allow for a natural filtering mechanism, where a user retransmits only subjects she is interested in. For a given configuration, we let $R(u)$ denote the set of subjects received by $u$. The utility $U_{u}(F)$ for user $u$ is the number of subjects in $S_{u}$ she receives, that is $U_{u}(F)=\left|R(u) \cap S_{u}\right|$. The utility of $u$ is maximized if $U_{u}(F)=\left|S_{u}\right|$.

We denote by move, a shift from a collection $F$ of strategies to a collection $F^{\prime}$ where a single user $u$ changes her strategy from a set $F_{u}$ to another $F_{u}^{\prime}$. (We say that $u$ rewires her connections.) The move is selfish if $U_{u}\left(F^{\prime}\right)>U_{u}(F)$. Selfish dynamics (or dynamics for short) are the sequences of selfish moves. We say that dynamics converge if any sequence of selfish moves is necessarily finite. The network is at equilibrium (or stable) if no selfish move is possible. In standard game-theoretic terminology, this corresponds to a pure Nash equilibrium. The global welfare of the system is defined as the overall system utility: $\mathcal{U}=\sum_{u \in V} U_{u}$. The efficiency of selfish, self-organization of a game is classically captured by the notion of price of anarchy defined as the ratio of the optimal global welfare over the global welfare of the worst equilibrium: PoA $=\frac{\max _{F \in \mathcal{F}} \sum_{u \in V} U_{u}(F)}{\min _{F \in \mathcal{E}} \sum_{u \in V} U_{u}(F)}$, where $\mathcal{F}$ denotes the set of possible collection of strategies and $\mathcal{E} \subseteq \mathcal{F}$ denotes the set of equilibria.

In some of our proofs we make use of the notion of potential functions. An ordinal (or general [9]) potential function [22] is a function $f: \mathcal{F} \rightarrow \mathbb{R}$ such that $\operatorname{sign}\left(f\left(F^{\prime}\right)-f(F)\right)=\operatorname{sign}\left(U_{u}\left(F^{\prime}\right)-U_{u}(F)\right)$ for any move from $F$ to $F^{\prime}$ where user $u$ changes her strategy. If $f\left(F^{\prime}\right)-f(F)=U_{u}\left(F^{\prime}\right)-$ $U_{u}(F), f$ is called an exact potential function. This notion was introduced by Monderer and Shapley [22] who show that it is tightly related to the notion of a congestion game [25]. The use of potential functions is a standard technique to show convergence of dynamics and to bound price of anarchy [9, 26].

\section{Homogeneous interests}

We first consider the case where identical sets of interests, $S_{u}=P$, for all $u \in V(G)$. In this context, we first analyze how to achieve optimal global welfare before establishing an upper bound on the price of anarchy. We 
will then show convergence of dynamics and provide a polynomial bound on convergence time.

\subsection{Optimal Utility and Optimal Global Welfare}

We first analyze what is the optimal utility, i.e. the maximum utility a user can get, and compare it to the optimal global welfare, i.e. the sum of user utilities obtained under an optimal centrally designed configuration.

First consider the maximum utility a given user $u$ can get. Clearly, $u$ cannot achieve utility larger than $p$, which corresponds to obtaining all the subjects in $P$. Moreover, he cannot obtain more subjects than the aggregate budget of attention of all users, that is $\sum_{u \in V(G)} \Delta_{u}=n \bar{\Delta}$, where $\bar{\Delta}$ is the average in-degree per node. More precisely, when a user $v$ receives subjects obtained by a user $w$, some link must connect $w$ to $v$ or to a user $v$ is connected to by some path. Overall, at least one link per user $w \neq u$ must be consumed with a user-to-user connection and cannot be used to retrieve a subject from a producer. In a configuration where $u$ receives a maximum number of subjects, his utility is thus at most $\left(\sum_{u \in V(G)} \Delta_{u}\right)-(n-1)=1+\sum_{u \in V(G)}\left(\Delta_{u}-1\right)$. Note that this bound is achieved in a singly linked chain configuration where users are placed along a chain ( $u$ being the last node) and where each user follows the previous user in the chain and use remaining connections to follow producers. We thus get the following claim.

Claim 1 In an homogeneous flow game with producers and $n$ users with average budget $\bar{\Delta}$, the optimal utility a given user can get among all configurations is $U^{*}=\min (p, 1+n(\bar{\Delta}-1))$.

On the other hand, all users can receive the same set of $\min (p, n(\bar{\Delta}-1)) \geq$ $U^{*}-1$ subjects in a ring configuration which consists in forming an oriented ring between users and connecting all remaining connections to pairwise distinct producers as depicted by Figure 1. This shows that the average utility a user gets at optimal global welfare equals the optimal utility $U^{*}$ up to one.

We will prove that optimal global welfare is generally obtained with a ring configuration. However, the singly linked chain can be optimal in some special cases. For example, in a flow game with $n=2$ users having budget 2, a singly linked chain achieves a global welfare of 5 compared to 4 for the ring. With three users, both configurations achieve a global welfare of 9 . More generally, we prove the following. 


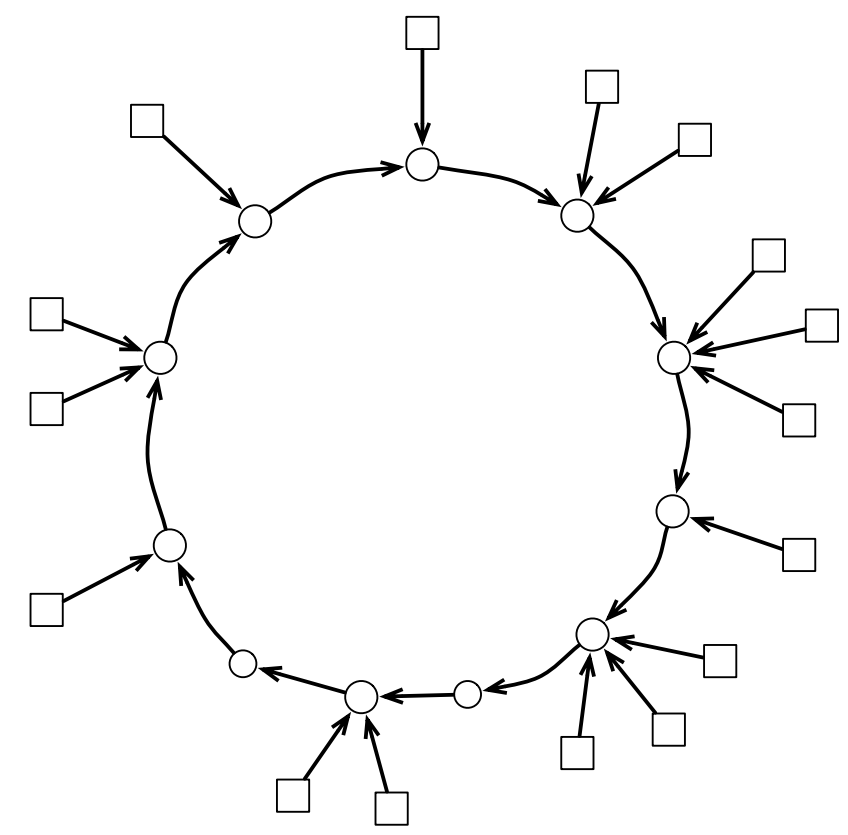

Figure 1: An optimal configuration in the homogeneous case (circles and squares represent users and producers respectively and edges point in the direction of content transfer, as in all figures throughout the paper). It is called a ring configuration as users form an oriented ring. 
Claim 2 Consider an homogeneous flow game with $n \geq 3$ users where each user has budget 2 or more and where the average budget $\bar{\Delta}$ is less than $\frac{p}{n}+1$ (equivalently $p \geq 1+n(\bar{\Delta}-1)$ ). The optimal global welfare is that of a ring configuration that is $n^{2}(\bar{\Delta}-1)=n\left(U^{*}-1\right)$.

Proof. The condition on average budget can be written $p>n(\bar{\Delta}-1)$. We first assume $p>n \bar{\Delta}$ as this implies that there always exists some producer not received by a user as $n \bar{\Delta}$ is a clear upper-bound of the number of subjects that can be globally retrieved by the users. Consider a configuration providing maximum global welfare.

The graph of user-to-user connections must be connected. If there were two different connected components $a$ and $b$, we could find a user $v$ in $b$ following some producer $x$. We could then rewire the producer-to-user connections in $a$ so that some node $u$ in $a$ receives $x$ plus some subject $y$ not received by $v$ while preserving the utility of each user (as nodes in $a$ have budget at least 2 , they receive at least two subjects). Rewiring $(x, v)$ into $(u, v)$ would then increase the global welfare of 1 at least.

Now consider the strongly connected components of this graph resulting from user-to-user connections: we call component a maximal set $W$ of users such that there is a path from $s$ to $t$ for all $s, t \in W$. Each component must be a singleton or a ring. This is because any strongly connected graph with a minimum number of links must be a ring (one link per user is necessary if the graph has two nodes or more, and it is sufficient only when these links form an oriented ring). If a non-singleton component was not a ring, we could rewire its internal connections to form a ring and use the saved connections to follow more producers and increase the global welfare.

We first show that if one component is a ring, then it is the only component. We show this by proving that there cannot exist a connection between a ring component and any other component. If a ring $b$ is connected to a ring $a$, then the two rings can be merged, allowing nodes in $a$ to receive subjects received in $b$ and not in $a$ previously. If a singleton component $\{v\}$ is connected to a ring $a$, then integrating $v$ in the ring allows all users in $a$ to receive the other subjects received by $v$. Finally, suppose a ring component $b$ is connected to a singleton component $\{u\}$ through a connection $(u, v)$ where $v$ is a node in $b$. As $u$ has budget at least 2 and connects to at most one component, it has at least one connection to a producer $x$. This connection can be used to integrate $u$ in the ring of $b$ while saving the previous ring connection of $v$. (If the node preceding $v$ in the ring is $w$, we replace $(x, u)$ 
by $(w, u)$ and release connection $(w, v)$.) We can thus connect $v$ to $x$ and get a configuration with higher welfare since $u$ now receives more subjects.

We now consider the special case where all components are singletons. If a user $a$ was followed by $b$ and $c$, we could increase the global welfare by letting $c$ follow $b$ instead of $a$. In such an optimal configuration, each user is followed by at most one user. The graph of user-to-user connections is thus a tree. Highest utility is obtained when the users follow pairwise disjoint sets of producers. If a node $a$ follows $b$ and $c$, it then more profitable to let a leaf of the sub-tree rooted at $b$ follow $c$ instead of some producer $x$ and let $a$ follow $x$ instead of $c$. The tree must thus be a singly linked chain. Such a configuration cannot achieve global welfare higher than that of a ring configuration for $n \geq 3$ and nodes of budget at least 2 .

Finally consider the case where $p>n(\bar{\Delta}-1)$ and $p \leq n \bar{\Delta}$. Consider the modified flow game with the same set of users (with the same budgets) and with $p^{\prime}=p+n>n \bar{\Delta}$ producers ( $n$ more producers are added). Any optimal configuration for the modified game can be transformed into a configuration for the original game since $n(\bar{\Delta}-1)<p$ producers at most get connected. The optimal configuration we consider in the original flow game must thus achieve a global welfare as high as what can achieved in the modified game. It is thus optimal in the modified game also. We can thus again conclude that the optimal global welfare is that of a ring, that is $n^{2}(\bar{\Delta}-1)=n\left(U^{*}-1\right)$.

Interestingly, we have indeed shown that for $n \geq 4$, the ring is the only optimal configuration when users have budget at least 2 . When some users have budget 1 and yet 4 or more nodes have budget at least 2, any optimal configuration still has a ring structure. As a connection to a budget 1 user is equivalent to a connection to the node he follows, we can virtually erase budget 1 users as transparent forwarding nodes along connections between users with budget at least 2 and producers. The virtual configuration must be optimal also and must be a ring. However, there are several ways budget 1 users can be connected to other users. They can be connected to any node of the ring, or form trees connected to some nodes of the ring, or form chains that replace some links of the ring. (A singly linked ring is one possible optimal configuration.) 


\subsection{Price of Anarchy}

We now consider a distributed setting where each user selfishly rewires his incoming connections if he can improve his utility, i.e., if this allows him to receive more subjects. The following proposition shows that with homogeneous user interests and budget of attention at least 3, self organization is efficient if dynamics converge, achieving a price of anarchy close to 1.

Proposition 1 Assume that $3 \leq \Delta_{u}<p$ for every user $u \in V$ of a homogeneous flow game with $n \geq 3$ users. Then under any equilibrium the utility of a user is at least $\frac{\bar{\Delta}-2}{\bar{\Delta}-1}\left(U^{*}-1\right)$ where $U^{*}$ is his optimal utility. The price of anarchy is $1+1 /(\bar{\Delta}-2)$ at most, approaching 1 for large $\bar{\Delta}$.

Before proving Proposition 1, we establish two lemmas. The first one allows to show the existence of strongly connected components at equilibrium showing that under some technical assumption, if a forwarding path exists at equilibrium, then a reverse path should also exists. .

Lemma 1 If an equilibrium is reached such that there exists a path $x, u_{1}, \ldots, u_{k}$ where $x$ is a producer, $u_{k}$ has in-degree bound $\Delta_{u_{k}} \geq 3$ and a producer $y$ is not received by $u_{k}$, then there is a path from $u_{k}$ to $u_{1}$.

Proof. The existence of the path $x, u_{1}, \ldots, u_{k}$ first implies that $R\left(u_{1}\right) \subset$ $R\left(u_{k}\right)$. Since $\Delta_{u_{k}} \geq 3, u_{k}$ must be connected to two nodes $v$ and $w$ distinct from $u_{k-1}$. We first claim that $v$ must bring at least one unique subject $z_{1}$ (not in $R\left(u_{1}\right)$ and thus different from $x$ ), otherwise, $u_{k}$ could unfollow $v$ and follow $y$ instead. Similarly, $w$ must bring at least one unique subject $z_{2}$ (different from $z_{1}$ and not in $R\left(u_{1}\right)$ ). Then if there is no path from $u_{k}$ to $u_{1}$, $u_{1}$ would unfollow $x$ and follow $u_{k}$ instead, so that he only loses one subject $x$ but gains at least two subjects $z_{1}$ and $z_{2}$.

The second Lemma will be used to bound the number of links between users in a strongly connected component at equilibrium. We call transitivity arc a link $(s, t)$ such that there exists a path from $s$ to $t$. Such a link is useless as any subjects it brings is also provided by the path, and node $t$ would be better off following a non-received producer instead of $s$. Such links cannot thus exist at equilibrium. 
Lemma 2 Consider a strongly connected graph $G$ with $n$ nodes and $m$ arcs (multiple arcs are allowed). If $m \geq 2 n-1$, then $G$ contains a transitivity arc.

Proof. We prove the result by induction on $n$. The hypothesis is true for $n=1$ (a self-loop on vertex $s$ is a transitivity arc for the empty directed path from $s$ to $s$ ). We denote by $n(G)$ the number of nodes in the graph $G$ and by $m(G)$ the number of edges in the graph $G$. Now consider $n>1$ and assume that the property is true for any graph $G^{\prime}$ with $n\left(G^{\prime}\right)<n$. Consider a strongly connected graph $G$ with $n$ nodes containing no transitivity arc. Since $n \geq 2, G$ must contain a circuit, i.e. an oriented cycle, with $k \geq 2$ nodes. The only arcs connecting two nodes of the circuit are the circuit arcs (otherwise, we would encounter a transitivity arc). Consider the graph $G^{\prime}$ obtained by contracting the circuit to one node. We have $m\left(G^{\prime}\right)=m(G)-k$ and $n\left(G^{\prime}\right)=n(G)-k+1<n$. Note that $G^{\prime}$ does not contain a transitivity arc either. Our induction hypothesis thus implies that $m\left(G^{\prime}\right)<2 n\left(G^{\prime}\right)-1$. That is $m(G)-k<2(n-k+1)-1$ or equivalently $m(G)<2 n-k+1 \leq 2 n-1$ as $k \geq 2$. The property is thus satisfied for $n$.

We are now ready to prove Proposition 1.

Proof.[of Proposition 1] Consider any equilibrium. If all users receive at least $p$ subjects, then the equilibrium is optimal. We thus consider the case where there is a user $u$ who receives less than $p$ subjects. Then $u$ must be connected to some producer $x$ by a path $x, u_{1}, \ldots, u_{k}=u$. Consider the graph $G^{\prime}$ induced by users reachable from $u_{1}$ that receive less than $p$ subjects. By Lemma 1, $G^{\prime}$ is strongly connected and all its users receive the same number $p^{\prime}<p$ of subjects.

We claim that two users $u$ and $v$ of $G^{\prime}$ cannot follow the same producer $y$. As there exists a path from $u$ to $v$, the link $(y, v)$ would be redundant and $v$ would be better off following some unreceived subject instead. Moreover, the fact that users in $G^{\prime}$ do not receive all subjects implies that they have spent all their budget of attention. We thus conclude that the number of edges in $G^{\prime}$ is $m\left(G^{\prime}\right)=\sum_{u \in V\left(G^{\prime}\right)} \Delta_{u}-p^{\prime}$. As the network is stable, there is no transitivity arc in $G^{\prime}$. (Otherwise, a transitivity arc $(s, t)$ would be redundant with some path from $s$ to $t$, and $t$ would be unstable as he could increase his utility by rewiring this link to a new producer.) Lemma 2 thus implies $m\left(G^{\prime}\right) \leq 2 n\left(G^{\prime}\right)-2 \leq 2 n\left(G^{\prime}\right)$, where $n\left(G^{\prime}\right)$ is the number of nodes in $G^{\prime}$. We thus get $p^{\prime} \geq \sum_{u \in V\left(G^{\prime}\right)} \Delta_{u}-2 n\left(G^{\prime}\right)=\sum_{u \in V\left(G^{\prime}\right)}\left(\Delta_{u}-2\right)$. 
First consider the case $p^{\prime} \leq p-2$. Suppose there exists a user $w \notin V\left(G^{\prime}\right)$. As $\Delta_{w} \geq 3, w$ has utility at least $p^{\prime}+2$ since he can gather the $p^{\prime}$ subjects received in $G^{\prime}$ plus two others by connecting to one node in $G^{\prime}$ plus the two corresponding producers. He thus receives two subjects not received in $G^{\prime}$ but this contradicts the stability as $u_{1}$ would better unfollow $x$ and connect to $w$. We thus conclude that $G^{\prime}$ indeed contains all users, implying $p^{\prime} \geq n(\bar{\Delta}-2)$. Using Claim [1, the utility of each user is at least $p^{\prime} \geq \frac{\bar{\Delta}-2}{\bar{\Delta}-1}\left(U^{*}-1\right)$. The global welfare at equilibrium is thus at least $n^{2}(\bar{\Delta}-2)$. As the optimal global welfare is at most $n^{2}(\bar{\Delta}-1)$ according to Claim 2 , the price of anarchy is at most $\frac{\bar{\Delta}-1}{\bar{\Delta}-2}$.

Finally, in the remaining case where $p^{\prime}=p-1$, some users may be outside $V\left(G^{\prime}\right)$. However such users must also receive $p-1$ subjects at equilibrium (if a user was receiving less, he could increase his utility by following a node in $\left.V\left(G^{\prime}\right)\right)$. The utility of each user is thus at least $\frac{p-1}{p} U^{*} \geq \frac{\bar{\Delta}-2}{\bar{\Delta}-1}\left(U^{*}-1\right)$ as $p \geq \bar{\Delta}-1$ and $U^{*} \geq\left(U^{*}-1\right)$. As the optimal global welfare is bounded by $n U^{*}$, the price of anarchy is at most $\frac{p}{p-1} \leq \frac{\bar{\Delta}-1}{\bar{\Delta}-2}$.

In both cases, each user gets utility $\frac{\bar{\Delta}-2}{\bar{\Delta}-1}\left(U^{*}-1\right)$ at least at equilibrium and the price of anarchy is at most $\frac{\bar{\Delta}-1}{\overline{\bar{\Delta}}-2}=1+\frac{1}{\bar{\Delta}-2}$.

Note that the above proposition is tight in the sense that high price of anarchy can arise when most of the users have budget only 2. Figure 2 presents the extreme configuration where all nodes have budget 2 . In this particular example, a doubly linked chain forms a pure Nash equilibrium gathering only two subjects in total while a ring configuration gathers $n$ subjects. The price of anarchy is thus $n / 2$. Indeed the doubly linked chain is still stable when some nodes have budget more than 2 and use their spare connections to gather fresh subjects. In that case, the price of anarchy is $\frac{n(\bar{\Delta}-1)}{n(\bar{\Delta}-2)+2}$. It thus remains unbounded as long as $\bar{\Delta}=2+o(1)$.

\subsection{Convergence of Dynamics}

We have thus shown that stable configurations of self-organizing networks with homogeneous user interests are efficient. However, do network dynamics converge to an equilibrium? The following proposition answers this question in the affirmative. 


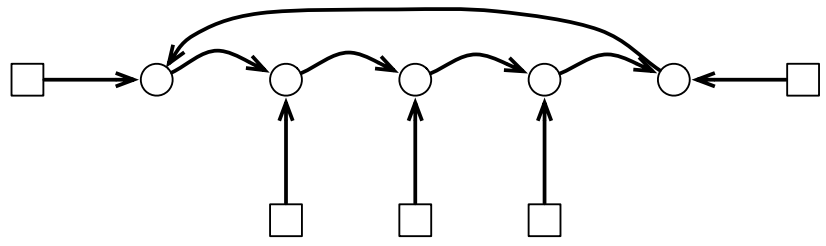

(a) Optimal configuration

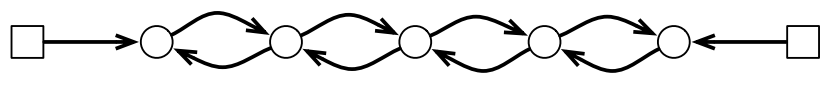

(b) A pure Nash equilibrium configuration

Figure 2: Two stable configurations in a homogeneous game where all users have degree $\Delta=2$ showing that price of anarchy can be $n / 2$.

Proposition 2 Any homogeneous flow game has an ordinal potential function, implying that selfish dynamics always converge to an equilibrium in finite time.

Proof. Let $n_{i}$ denote the number of users that receive $i$ subjects and consider the sequence $\left(n_{0}, n_{1}, \ldots, n_{p}\right)$. We show that this sequence always decreases according to lexicographic ordering when users make selfish moves. The function $-\sum_{0 \leq i \leq p} n_{i} n^{p-i}$ (obtained by reading $n_{0} n_{1} \cdots n_{p}$ as a number) is thus a potential function that will always increase until a local maximum is reached, proving convergence to an equilibrium.

Consider a user $u$ that is receiving $i$ subjects and that will make a selfish move to receive $j>i$ subjects instead. Note that there is no path from $u$ to any other user receiving $k<i$ subjects. Therefore any change by $u$ will not affect these users. Now consider any user $v$ with $k \geq i$ subjects. If there is no path from $u$ to $v$ then $u$ 's selfish move does not affect $v$. If there is such a path, then $v$ will now receive at least $j>i$ subjects. We thus now have $n_{i}-1$ users receiving $i$ subjects, and the sequence $\left(n_{0}, n_{1}, \ldots, n_{p}\right)$ has decreased according to lexicographic ordering.

Combining Proposition 1 1 and Proposition 2, we obtain:

Theorem 1 In a homogeneous flow game where $n \geq 3$ users have budget of attention at least 3 , less than $p$, and $\bar{\Delta}$ in average, selfish dynamics converge 
to an equilibrium such that the utility of a user is at least $\frac{\bar{\Delta}-2}{\bar{\Delta}-1}\left(U^{*}-1\right)$ where $U^{*}$ is the optimal utility he can get. The price of anarchy is $1+1 /(\bar{\Delta}-2)$ at most.

Our proof of Proposition 2 yields a very loose bound of $n^{p+1}$ on convergence time. We leave as an open question whether exponential time of convergence can really arise. However, in the following proposition we show that a homogeneous flow game with at least 4 subjects, a user with budget of attention at least 2 and a user with budget of attention at least 3 , is not equivalent to a congestion game. This rules out the possibility of using techniques similar to [9] to find equilibria in polynomial time, and more generally to easily bound convergence time.

Proposition 3 Any homogeneous flow game with at least 4 subjects, a user with budget of attention at least 2 and a user with budget of attention at least 3, does not admit an exact potential function.

Note that a game is equivalent to a congestion game if and only if it admits an exact potential function [22].

Proof. This is proven by considering cycles in the strategy space where each point corresponds to a set of strategies chosen by all the users and an arc corresponds to a selfish move by a user. A potential function assigns a value to each point. Its variation along an arc is the difference between the values assigned to the destination and the source. We define its variation along a path as the sum of the variations of the arcs of the path. Obviously, the variation along a cycle must be zero.

Additionally, an exact potential function should ensure that the variation during a move by a user $u$ equals the variation of the utility of $u$. We define similarly the variation of utility along a path with selfish moves from users $u_{1}, \ldots, u_{k}$ respectively as the sum of utility variations for node $u_{1}$ in the first move, node $u_{2}$ in the second move, and so on. If ever, we can exhibit a cycle with non-zero utility variation in our flow game, it is clearly impossible to design an exact potential function for that game satisfying both requirements. (For more details about congestion games and exact potential functions, see for example [22].)

To show the proposition, we exhibit a 4-cycle in the strategy space with non-zero utility variation along the cycle. Without loss of generality, the game contains four producers $\{a, b, c, d\}$ and two users $u, v$ with $\Delta_{u} \geq 2$ 
and $\Delta_{v} \geq 3$ as depicted in Figure 3. User $u$ can adopt in particular strategy $A=\{(a, u)\}$ or $B=\{(b, u),(c, u)\}$. User $v$ can adopt in particular strategy $C=\{(u, v),(b, v),(c, v)\}$ or $D=\{(u, v),(d, v)\}$. Consider the cycle $(A, C) \rightarrow(B, C) \rightarrow(B, D) \rightarrow(A, D) \rightarrow(A, C)$ where user $u$ moves from strategy $A$ to $B$ increasing his utility by 1 , then $v$ moves from $C$ to $D$ and increases his utility by 1 , then $u$ moves back to $A$ with a utility variation of -1 , and finally $v$ moves back to $C$ increasing its utility by 1 again (the strategies for other users remain fixed). The overall sum is thus $2 \neq 0$.

Instead of trying to obtain tight bounds on the convergence time of arbitrary sequences of moves, we will now prove that convergence time is polynomial under some natural assumption concerning the dynamics. The idea is to assume some fairness among users in the sense that they regularly have the opportunity to make a move. To measure this, we call round a sequence of moves where each user can be associated to a point in the sequence where he either performs a selfish move or cannot make any selfish move (if he is given the possibility to make a move at that point, he can either perform the move indicated in the sequence or no move can increase his utility in the configuration obtained by the moves up to that point). Interestingly, we could consider a sequence of moves as fair when starting at any moment $t$ in the sequence, the moves from $t$ to $t+O(n)$ constitute a round. If convergence is polynomial in number of rounds, it is then polynomial in number of moves also. More generally, we consider as fair any sequence of moves without any infinite round. Such sequences will be called fair dynamics and can always be decomposed as a sequence of finite rounds.

Proposition 4 Any homogeneous flow game with p producers and $n$ users having average budget of attention $\bar{\Delta}$ converges in $O\left(n p+n^{2} \bar{\Delta}\right)$ rounds under fair dynamics.

Proof. We claim that the number of users with minimum utility $U_{m}$ (those getting the least number $U_{m}$ of subjects) decreases every three rounds as long as equilibrium is not reached and the value of $U_{m}$ has not increased. The proposition clearly follows from that fact since $3 n$ rounds at most then suffice to increase the minimum utility by one at least and the maximum utility a user can get is bounded by $\min (p, n \bar{\Delta})$. To show this, assume that during one round, no user with minimum utility can make a selfish move. A first trivial case occurs when all users have the same utility $U_{m}$. This 


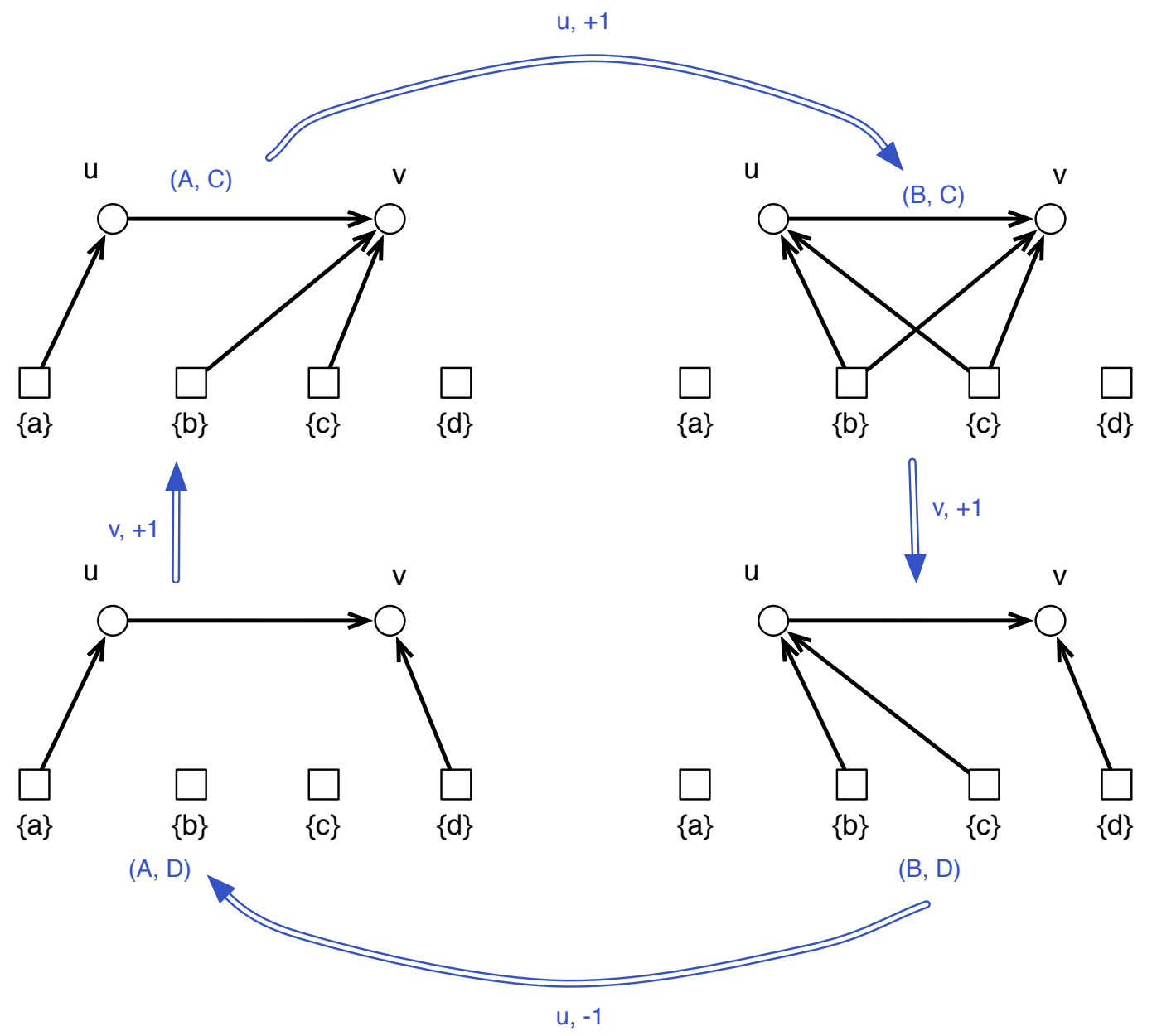

Figure 3: A 4-cycle $(A, C) \rightarrow(B, C) \rightarrow(B, D) \rightarrow(A, D) \rightarrow(A, C)$ in the strategy space with non-zero utility variation. The cycle is represented with blue double arrows. Each double arrow corresponds to a move where a user changes his connection strategy. This brings the network from one configuration to another. Each double arrow is labeled with the name of the user making the move and his utility variation. 
means that no user could make a move which implies that equilibrium has been reached. Otherwise, consider what occurs in the next round. Either no node can move and we reach equilibrium, or a node with minimum utility $U_{m}$ can move, or a node $u$ receiving more than $U_{m}$ subjects can move and then receives at least $U_{m}+2$ subjects. In the latter case, consider the first time the opportunity of moving is given to a node $v$ receiving $U_{m}$ subjects and following directly at least one producer $x$. (Such a node must exist: as any user followed by a user having minimum utility has also minimum utility, if all users with minimum utility are only connected together, their utility is zero and they can obviously move.) Then rewiring $(x, v)$ into $(u, v)$ is a move for $v$. This is due to the fact that $u$ still receives $U_{m}+2$ subjects at least until the turn of $v$ comes (any move by a user $u^{\prime}$ with utility $U_{m}+1$ or more increases his utility to $U^{\prime} \geq U_{m}+2$ and if $u$ is affected, his utility cannot drop bellow $U^{\prime}$ ). As $u$ receives at least two subjects not received by $v$, the utility of $v$ increases by one at least with this move. As the move of $u$ occurs within the second round after a round without any progress for nodes with minimum utility, the move of $v$ occurs within the third round at most.

The interested reader can easily build sequences of moves with length $\Omega\left(n^{2}\right)$. We will thus not try to improve beyond polynomial time convergence in this section. However, we will see in the heterogeneous case how convergence within a logarithmic number of rounds can arise when interests of users have some geometrical structure (see Section 5).

\section{Heterogeneous interests}

We now consider the more realistic case where users have differing sets of interests. To make the model even more general, we assume here that users weight independently topics. Let $W_{u}(s)$ denote the weight (or value) of topic $s$ to user $u$. The objective of a user is now to maximize the sum of the values of subjects he receives. We will consider user-interest sets $S_{u} \subseteq P$ that include topics of non-zero value, that is $S_{u}=\left\{s: W_{u}(s)>0\right\}$. Such user-specific weights for topics represent a natural expertise or focussed interest users may have on a subset of topics. (Note that the model presented previously corresponds to $W_{u}(s)=1$ for $s \in S_{u}, W_{u}(s)=0$ for $s \notin S_{u}$.) 


\subsection{Price of Anarchy}

We now show that the price of anarchy of such a system may be unbounded.

Proposition 5 In a heterogeneous flow game with $n$ users having budget of attention $\Delta$ each, the price of anarchy can be $\Omega\left(\frac{n}{\Delta}\right)$.

Proof. We show the result through an example, illustrated in Figure 4 , For positive integer $k$, consider a system with $n=2 k$ users having budget of attention $\Delta \geq 2$ each, and $p=2(\Delta-1) k$ producers. We distinguish two set of users $\left\{a_{1}, \ldots, a_{k}\right\}$ and $\left\{b_{1}, \ldots, b_{k}\right\}$. Similarly, the producers are partitionned into groups $\left\{A_{1}, \ldots, A_{k}\right\}$ and $\left\{B_{1}, \ldots, B_{k}\right\}$ where each $A_{i}$ (resp. $\left.B_{i}\right)$ contains $\Delta-1$ producers.

As illustrated in Figure 4(a), each user $a_{i}$ has a value of 1 for each topic in $A_{i} \cup B_{i}$ and additionally the first element of each $A_{j}$ for $j \neq i$. In the figure, this is represented by the solid red line. Similarly, each user $b_{i}$ has a value of 1 for each topic in $A_{i} \cup B_{i}$ and additionally the first element of each $B_{j}$ for $j \neq i$. This is represented by the dashed blue line in the figure. Users have a value of zero for all other topics.

Figure 4(b) shows a benchmark configuration, with solid red edges for nodes of type $a$ and dashed blue edges for nodes of type $b$. In this configuration, users $a_{i}, i=1, \ldots, k$ construct an oriented ring, and similarly users $b_{i}, i=1, \ldots, k$ construct a separate oriented ring. They use their remaining links to connect to producers. User $a_{i}$ is then connected to $a_{i-1}$ (with $a_{0}$ corresponding to $a_{k}$ ) and also to all producers in $A_{i}$ using the remaining $\Delta-1$ links. Similarly, user $b_{i}$ is connected to $b_{i-1}$ (with $b_{0}$ corresponding to $b_{k}$ ) and also to all producers in $B_{i}$. The corresponding utility is $n(n / 2+\Delta-2)$, so that the optimal global welfare $\mathcal{U}^{*}$ satisfies $\mathcal{U}^{*} \geq n^{2} / 2$.

Figure 4(c) shows an equilibrium configuration, where each user $a_{i}$ (resp. $b_{i}$ ) connects to producers in $A_{i}$ (resp. $\left.B_{i}\right)$ using $\Delta-1$ links and to $b_{i}$ (resp. $a_{i}$ ) using one link. Note that neither user can gain by making a unilateral move since each of the other users (of indices $j \neq i$ ) can only provide one additional subject as opposed to the $\Delta-1$ subjects they now receive from each other. The global utility here is $\mathcal{U}=n(2 \Delta-2) \leq 2 n \Delta$, and the price of anarchy is thus at least $\frac{n}{4 \Delta}$. 

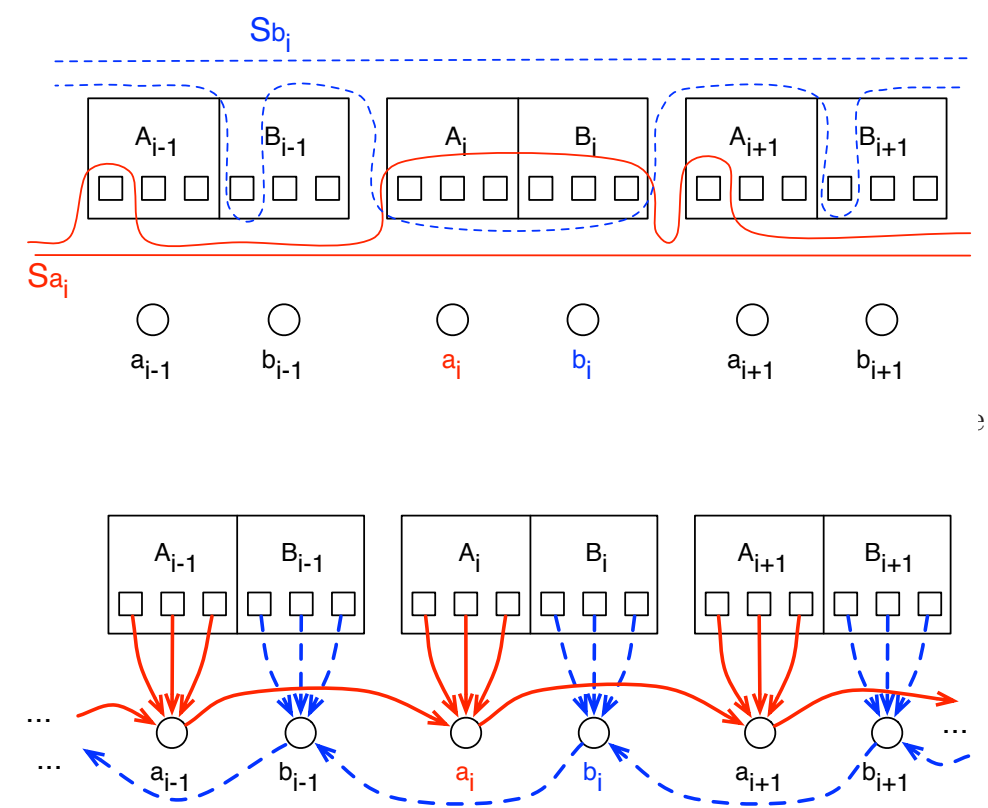

(b) Benchmark configuration with two oriented rings.
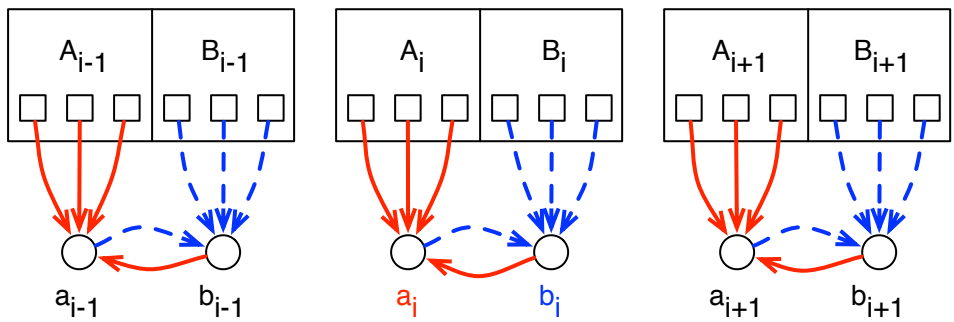

(c) A pure Nash equilibrium configuration.

Figure 4: A heterogeneous flow game where all users have same budget of attention $\Delta=4$ and two configurations showing that price of anarchy can reach $\frac{n}{4 \Delta}$. 


\subsection{Convergence of dynamics}

We have shown that the price of anarchy can be unbounded with respect to the number of users in some cases.

We now show that selfish dynamics do not even guarantee convergence to an equilibrium.

Proposition 6 Selfish dynamics of a flow game with heterogeneous utilities may not converge.

Proof. Consider the following scenario with six retransmitting users $p_{i}, q_{i}, r_{i}$, $i=1,2$, and two users $u_{1}, u_{2}$ each with degree $\Delta_{i}=3$. The retransmitting users publish sets of topics as follows: $p_{1}:\{a, b\}, p_{2}:\{c, d\}, q_{1}:\{x, y\}$, $r_{1}:\{k, l\}, q_{2}:\{x, k\}, r_{2}:\{y, l\}$. The user-specific values are given in Table 1, where $\epsilon<1 / 2$. As depicted in Figure 5, each agent $u_{i}$ uses one connection to follow user $p_{i}$ through whom he receives a total value of 4 . He also connect to the other user $u_{3-i}$ to receive another topic of value 2 from $p_{3-i}$. Now each user $u_{i}$ must select between $q_{1}, q_{2}, r_{1}$ and $r_{2}$ for his third connection. We start with users $u_{1}$ and $u_{2}$ choosing $q_{1}$ and $q_{2}$ respectively. They thus receive $8+\epsilon$ and $7+2 \epsilon$ in total respectively. User $u_{2}$ then selects $r_{2}$ for receiving $l$ instead of $k$ and getting $8+\epsilon$. This changes user $u_{1}$ 's utility to $7+2 \epsilon$. Then user $u_{1}$ can increase his utility by $1-\epsilon$, and does so by switching to $r_{1}$ for receiving $k$ instead of $x$. Now this decreases $u_{2}$ 's utility by $1-\epsilon$. This can indeed continue again and again as follows. Denote the state of the system by $\left(\mathcal{S}\left(u_{1}\right), \mathcal{S}\left(u_{2}\right)\right)$ where $\mathcal{S}\left(u_{i}\right)$ is user $u_{i}$ 's strategy in selecting between $q_{i}$ and $r_{i}$. Under selfish moves, the system may cycle as follows: $\left(q_{1}, q_{2}\right) \rightarrow\left(q_{1}, r_{2}\right) \rightarrow\left(r_{1}, r_{2}\right) \rightarrow\left(r_{1}, q_{2}\right) \rightarrow\left(q_{1}, q_{2}\right) \rightarrow\left(q_{1}, r_{2}\right) \rightarrow \cdots$.

\begin{tabular}{|l|l|l|l|l|l|l|l|l|}
\hline User $\backslash$ Topic & $a$ & $b$ & $c$ & $d$ & $x$ & $y$ & $k$ & $l$ \\
\hline$u_{1}$ & 2 & 2 & 2 & 0 & $\epsilon$ & 1 & 1 & $\epsilon$ \\
\hline$u_{2}$ & 2 & 0 & 2 & 2 & 1 & $\epsilon$ & $\epsilon$ & 1 \\
\hline
\end{tabular}

Table 1: User-specific values for topics used in the proof of Proposition 6 ,

With an arbitrary structure of user interest sets, we have thus shown that the price of anarchy may be unbounded, and dynamics may not converge. The question of determining if pure Nash equilibria exist is left open. 


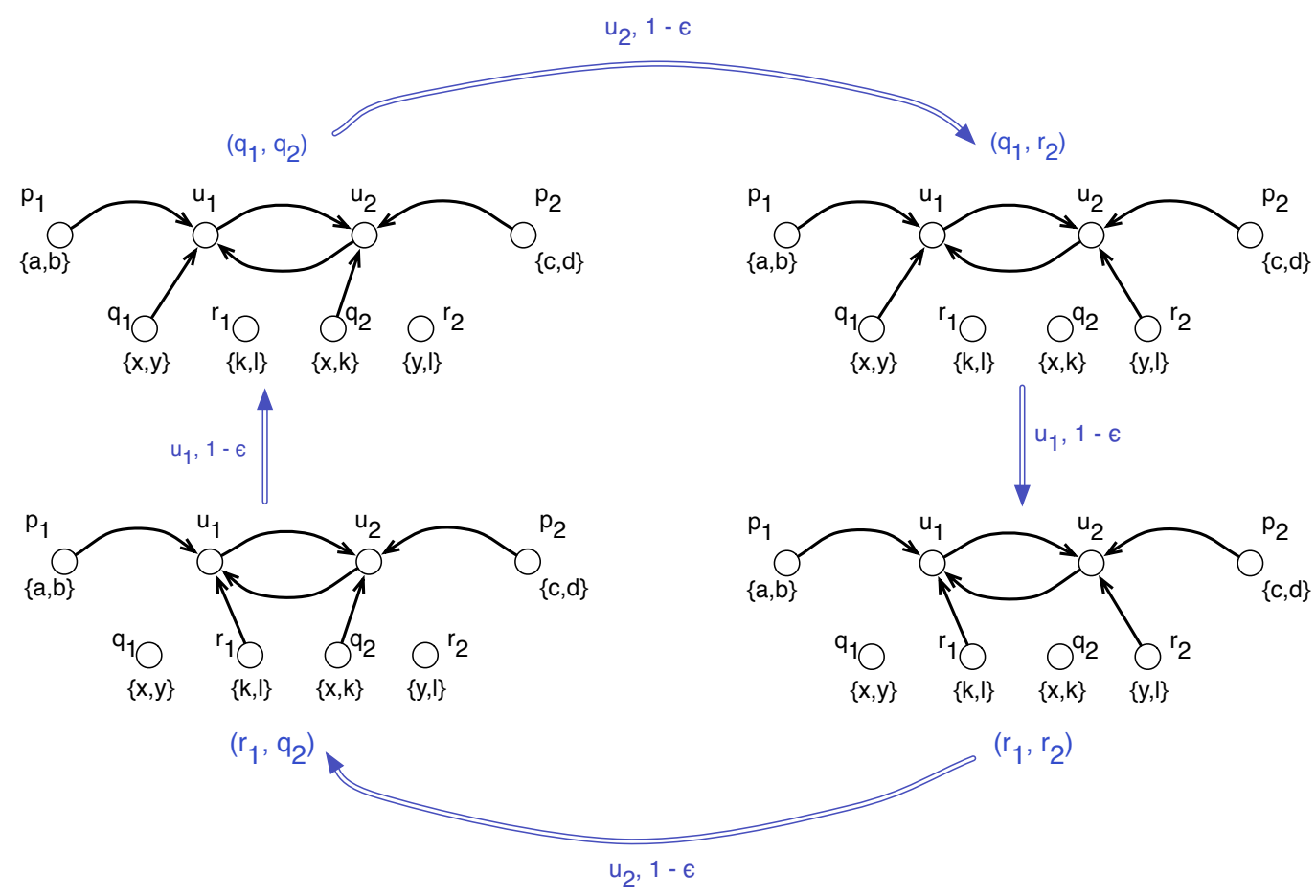

Figure 5: Illustration of the proof of Proposition 6. Instability with heterogeneous interest sets can arise with this 4-cycle of selfish moves $\left(q_{1}, q_{2}\right) \rightarrow\left(q_{1}, r_{2}\right) \rightarrow\left(r_{1}, r_{2}\right)$ $\rightarrow\left(r_{1}, q_{2}\right)$ where users $u_{1}$ and $u_{2}$ change only one connection. The strategy of other users remains fixed. The set of subjects they receive is indicated. User-specific values for topics are those listed in Table 1. Each double arrow corresponds to a selfish move bringing from one configuration to another. It is labeled with the name of the user making the move and his utility variation. 


\section{Structured interest sets}

We now revisit the efficiency of social filtering in an heterogeneous scenario, where interest sets are no longer arbitrary but instead are organized according to a well behaved geometry. Specifically we assume the following model. A metric $d$ is given on a set $P^{\prime} \supseteq P$ of subjects. The interest set $S_{u}$ of each user $u$ then coincides with a ball $B\left(s_{u}, R_{u}\right)$ in this metric, specified by a central subject $s_{u}$ and a radius of interest $R_{u}$. We assume that the value of a subject to a user is non-increasing in its distance from $s_{u}$. Specifically, we assume $W_{u}(s)=f\left(d\left(s_{u}, s\right)\right)$ for $d\left(s_{u}, s\right) \leq R_{u}$, where $f(\cdot)$ is a non-increasing positive function, and $W_{u}(s)=0$ otherwise. Without loss of generality, we can assume $P^{\prime}=\left\{s_{u}: u \in V\right\} \cup P$ and $S_{u}=B\left(s_{u}, R_{u}\right) \cap P$. We shall first give conditions on the metric $d$ and the sets $S_{u}$ under which an efficient configuration exists. We will then introduce modified dynamics and filtering rules which guarantee stability, i.e. convergence to an equilibrium. A flow game where interest sets can be defined in this way is called a metric flow game.

The model can easily be generalized to more eclectic user interests where topics a user is interested in correspond to the union of a constant number of balls. We leave out the details of such generalizations so as to keep the focus of the paper. However, we include a brief discussion later in the section, in the context of Proposition 7.

\subsection{Sufficient conditions for optimal utility}

Consider the following properties of the interest set geometry.

1. $\gamma$-doubling: $d$ is $\gamma$-doubling, i.e. for any subject $s$ and radius $R$, the ball $B(s, R)$ can be covered by $\gamma$ balls of radius $R / 2$ : there exists $I \subset S$ such that $|I| \leq \gamma$ and $B(s, R) \subset \cup_{t \in I} B(t, R / 2)$.

2. $r$-covering: $r$ is a covering radius, i.e. for each subject $s \in P$ there is a user $u$ such that $d\left(s_{u}, s\right) \leq r$ and $R_{u} \geq r$.

3. $(r, \delta)$-sparsity: there are at most $\delta$ subjects within distance $r:|B(s, r)| \leq$ $\delta, \forall s$.

4. $r$-interest-radius smoothness: for any users $u, v$ with $d\left(s_{u}, s_{v}\right)<3 R_{u} / 2+$ $r$, we have $R_{v} \geq R_{u} / 2+r$ and $R_{u} \geq R_{v} / 2+r$ (users with similar interests have comparable interest radii). 
Property (11) is a classical generalization of dimension from Euclidean geometry to abstract metric spaces (an Euclidean space with dimension $k$ is $2^{\Theta(k)}$-doubling). This is a natural assumption if user interests can be modeled by proximity in a hidden low-dimensional space. Property (2) states that all subjects are within distance $r$ from some user's center of interest and can thus be seen as an assumption of minimum density of users' interests over the whole set $P$ of available subjects. Property (3) puts an upper bound on the density of subjects. In other words, we assume a level of granularity under which we do not distinguish subjects. Property (4) is another form of smoothness assumption, requiring that the radii of interest of nearby users do not differ too much. This property is obviously satisfied if we assume that all users have same radius of interest. In general, it may seems debatable if we think of an expert next to an amateur. However, if we assume that a topic is split into several subjects according to the level of expertise required to understand the corresponding news, the assumption becomes more natural as an expert is still interested in related subjects (with lower level of understanding) and an amateur still has some focus if the correct number of levels is considered.

We now show that an optimal solution exists, i.e. one in which each user receives all subjects in his interest set, as each user $u$ has budget of attention at least $\gamma \delta+\gamma^{2} \log \frac{R_{u}}{r}$.

Proposition 7 Consider a metric flow game satisfying the $\gamma$-doubling, $r$ covering, $(r, \delta)$-sparsity and $r$-interest-radius smoothness assumptions. If in addition each user $u$ has a budget of attention at least $\gamma \delta+\gamma^{2} \log \frac{R_{u}}{r}$, then there exists a collection of user strategies allowing each user $u$ to receive all subjects in $S_{u}$.

This result can easily be extended to the case where each user interest set is given by a union of balls (the number of balls being at most a constant $b$ ). It suffices to repeat the construction of the proof for each ball, resulting in a factor $b$ in the resulting required budget of attention. The assumptions have to be slightly modified so that any subject is covered by some ball of a user (in the covering assumption) and that two nearby balls have comparable radii (in the smoothness assumption).

Proof. We define the ball $B_{u, i}:=B\left(s_{u}, \min \left(R_{u}, 2^{i} r\right)\right)$ for each user $u$ and each integer $i \geq 0$. The construction to follow will ensure that $u$ collects all subjects in $B_{u, i}$ through a set $N_{u, i}$ of contacts such that $B_{u, i} \subset \cup_{v \in N_{u, i}} B_{v, i-1}$. 
We first define $N_{u, 1}=\left\{p_{s}: s \in B_{u, 1}\right\}$. According to the $\gamma$-doubling assumption, $B_{u, 1}$ can be covered by $\gamma$ balls of radius $r$. As the $(r, \delta)$-sparsity implies that each of these balls contains $\delta$ subjects at most, the size of $N_{u, 1}$ is upper bounded by $\gamma \delta$.

Now, for $2 \leq i \leq\left\lceil\log \frac{R_{u}}{r}\right\rceil$, the $\gamma$-doubling assumption implies that $B_{u, i}$ can be covered by at most $\gamma^{2}$ balls of radius $2^{i-2} r$ : there exists a set $L_{u, i}$ of at most $\gamma^{2}$ subjects such that $B_{u, i} \subset \cup_{s \in L_{u, i}} B\left(s, 2^{i-2} r\right)$. From the $r$-covering assumption, we can then define a set $N_{u, i}$ of at most $\gamma^{2}$ users such that each $s \in L_{u, i}$ is at distance at most $r$ from some $s_{v}$ with $v \in N_{u, i}$. We then have $B_{u, i} \subset \cup_{v \in N_{u, i}} B\left(s_{v}, 2^{i-2} r+r\right)$. Without loss of generality, we can assume that for each $s \in L_{u, i}, B\left(s, 2^{i-2} r\right)$ intersects $B_{u, i}$ (otherwise $s$ can safely be removed from $L_{u, i}$ as it does not cover anything useful). We thus have $d\left(s_{u}, s\right) \leq R_{u}+2^{i-2} r<3 R_{u} / 2$ (note that $2^{i-1} r<R_{u}$ as $i \leq\left\lceil\log \frac{R_{u}}{r}\right\rceil$ ). For $v \in N_{u, i}$ such that $d\left(s, s_{v}\right) \leq r$, we then have $d\left(s_{u}, s_{v}\right)<3 R_{u} / 2+r$. From the $r$-interest-radius smoothness, we then deduce $R_{v} \geq R_{u} / 2+r>$ $2^{i-2} r+r$, implying $\min \left(R_{v}, 2^{i-1} r\right) \geq 2^{i-2} r+r$. The ball $B_{v, i-1}$ thus contains $B\left(s_{v}, 2^{i-2} r+r\right) \supset B\left(s, 2^{i-2} r\right)$. Together with the definition of $L_{u, i}$, this proves $B_{u, i} \subset \cup_{v \in N_{u, i}} B_{v, i-1}$.

The connection graph $G$ results from connecting each user $u$ to all contacts in the set $\cup_{1 \leq i \leq\left\lceil\log \frac{R_{u}}{r}\right\rceil} N_{u, i}$.

Flow correctness: We show by induction on $i$ that each user $u$ receives all subjects in $B_{u, i}$. The direct connection to producers for subjects in $B_{u, 1}$ ensures this for $i=1$. For $i>1$, the induction hypothesis implies that each user $v \in N_{u, i}$ receives all subjects in $B_{v, i-1}$. From $B_{u, i} \subset \cup_{v \in N_{u, i}} B_{v, i-1}$, we conclude that $u$ will receive news about subjects in $B_{u, i}$ from its contacts in $N_{u, i}$. As $S_{u}=B_{u,\left\lceil\log \frac{R_{u}}{r}\right\rceil}$, we finally know that $u$ receives all subjects in $S_{u}$.

In-degree bound: First, we have $\left|N_{u, 1}\right| \leq \gamma \delta$. This comes from the fact that $B_{u, 1}$ is included in at most $\gamma$ balls of radius $r$ from the $\gamma$-doubling assumption, and each of these balls contains at most $\delta$ subjects from the $(r, \delta)$-sparsity assumption. Second, we have already seen that $\left|N_{u, i}\right| \leq \gamma^{2}$ for $2 \leq i \leq\left\lceil\log \frac{R_{u}}{r}\right\rceil$. We thus obtain the bound $\gamma \delta+\gamma^{2}\left(\left\lceil\log \frac{R_{u}}{r}\right\rceil-1\right)<$ $\gamma \delta+\gamma^{2} \log \frac{R_{u}}{r}$.

The core of the construction consists in covering a given ball radius of $2^{i} r$ with a set of $\gamma$ balls of radius $2^{i-1} r$. As a covering set of $\gamma^{2}$ balls can 
be computed through a simple greedy covering algorithm [13, a solution where the required budget of attention is within a factor $\gamma$ from the bound of Proposition 7 can thus be computed in polynomial time.

Note that a logarithmic number of connections allows to gather a polynomial number of subjects. As previously mentioned, a budget of attention

of $\Delta=\gamma \delta+\gamma^{2} \log \frac{R_{u}}{r}$ for each user $u$ is enough for maximum utility. On the other hand, the number of subjects in $B\left(s_{u}, R_{u}\right)$ can be polynomial in $R_{u}$. For example, if the subjects are placed regularly in a $d$ dimensional lattice, it would be in the order of $R_{u}^{d}$ (the doubling assumption ensures that it is at most polynomial). A logarithmic number of connections is thus sufficient for gathering the subjects interesting a user. Thus this configuration gives substantial savings in comparison to one where users would connect directly to all their subjects.

Clearly the configuration graph identified in this theorem is an equilibrium: as maximum utility is reached, no user can increase its utility by reconnecting. We now study conditions that guarantee convergence of dynamics.

\subsection{Sufficient conditions for stability}

We first define two rules regarding republication of subjects received and reconnections.

1. Expertise-filtering rule: when a user $u$ is connected to a user $v, u$ only receives subjects $s$ such that $d\left(s_{v}, s\right) \leq d\left(s_{u}, s\right)$.

2. Nearest-subject rule for re-connection: when reconnecting, each user $u$ gives priority to subjects that are closer to $s_{u}$ : a new subject $s$ is gained by $u$ so that no subject $t$ with $d\left(s_{u}, t\right) \leq d\left(s_{u}, s\right)$ is lost. (On the other hand, any subject $t$ with $d\left(s_{u}, t\right)>d\left(s_{u}, s\right)$ can be lost.)

Rule 1 can be interpreted as follows. The center of expertise of a user is the same as its center of interest, and the distance $d$ also captures expertise of users about subjects, in that $u$ is more expert than $v$ on subject $s$ if and only if $d\left(s_{u}, s\right) \leq d\left(s_{v}, s\right)$. The rule then amounts to a sanity check where $u$ discards news from sources that have less expertise than himself on the subject. We capture this with the following slight variation of the model. A flow game with expertise-filtering is a flow game where reception of a subject $s$ by user $u$ occurs only when there exists a directed path $s=u_{0}, \ldots, u_{k}=u$ 
from $s$ to $u$ such that for each $1 \leq i<k, s \in S_{u_{i}}$ (i.e. $\left.d\left(s_{u_{i}}, s\right) \leq R_{u_{i}}\right)$ and $d\left(s_{u_{i}}, s\right) \leq d\left(s_{u_{i+1}}, s\right)$.

Rule2 states that a user $u$ prefers to receive a subject he is more interested in (i.e. closer to $s_{u}$ ) rather than any number of subjects that are less interesting. A flow game is denoted to be with nearest-subject priority if the utility function of each user $u$ is defined by $U_{u}(F)=\max \left\{R: u\right.$ receives all $\left.s \in B\left(s_{u}, R\right)\right\}$.

Proposition 8 Any metric flow game with expertise-filtering and nearestsubject priority has an ordinal potential function, implying that selfish $d y$ namics always converge to an equilibrium in finite time.

The proof shows the existence of an ordinal potential function.

Proof. Consider the set $\mathcal{D}=\left\{d\left(s_{u}, s\right): u \in V, s \in P\right\}$ of all possible distances from the central subject of any user to any subject. Let $r_{1}, \ldots, r_{m}$ denote all elements of $\mathcal{D}$ sorted in non-decreasing order (i.e. $r_{1} \leq \cdots \leq r_{m}$ ) with ties broken arbitrarily. Let $n_{i}$ denote the number of pairs $(u, s)$ such that $d\left(s_{u}, s\right)=r_{i}$ and $u$ receives $s$. Consider the tuple $\left(n_{1}, \ldots, n_{m}\right)$. When a user $u$ makes a selfish move, he increases his utility by receiving a new subject $s$. Let $i$ denote the index such that $d\left(s_{u}, s\right)=r_{i}$. Any lost subject $t$ must satisfy $d\left(s_{u}, t\right)>d\left(s_{u}, s\right)$ by the nearest-subject rule. If a lost subject $t$ was received by some user $v$ through a path from $u$ to $v$, we have $d\left(s_{v}, t\right) \geq d\left(s_{u}, t\right)$ by the expertise-filtering rule. We thus deduce $d\left(s_{v}, t\right)>d\left(s_{u}, s\right)$, implying that $n_{j}$ can decrease only for $j>i$. The tuple $\left(n_{1}, \ldots, n_{m}\right)$ thus increases according to the lexicographical order after any selfish move. As the size of $\mathcal{D}$ is at most $n p$, the product $n p$ is also a trivial upper bound on each $n_{i}$. The tuple $\left(n_{1}, \ldots, n_{m}\right)$ can thus be read as a number in base $n p$. This number always increases under selfish moves, implying that $\sum_{0 \leq i \leq m} n_{i}(n p)^{m-i}$ is a potential function. This potential function always increases until a local maximum is reached, proving convergence to an equilibrium.

We can additionally prove fast convergence under sufficient conditions for optimal utility and fair dynamics under best response. We call best response a move where a user $u$ gets the best possible utility (with nearest-subject priority) given the current connections of other users. In other words, $u$ receives all subjects within distance $R$ from $s_{u}$ after the move and no move could provide all subjects within distance $R^{\prime}$ from $s_{u}$ with $R^{\prime}>R$. Recall that a sequence is fair if it can be decomposed in a sequence of rounds where each user has the opportunity to make a move during each round as defined 
in Section 3.3. If in addition users only perform best response moves, we say that the system is under fair dynamics with best response.

We are now ready to prove the following:

Theorem 2 Consider a metric flow game with expertise-filtering and nearestsubject priority that satisfies the $\gamma$-doubling, $r$-covering, $(r, \delta)$-sparsity and $r$ interest-radius smoothness assumptions. If in addition each user $u$ has budget of attention at least $\gamma \delta+\gamma^{2} \log \frac{R_{u}}{r}$, selfish dynamics converge to an equilibrium where each user $u$ receives all subjects in $S_{u}$, implying that the price of anarchy is then 1. Moreover, the system converges in at most $\left\lceil\log \frac{R_{m}}{r}\right\rceil$ rounds under fair dynamics with best response where $R_{m}$ is the maximum radius of interest over all users.

Proof. Consider a configuration where some user $u$ does not receive some subject $s$ in his interest ball. Such a pair $(u, s)$ is called an unsatisfied pair. Without loss of generality we consider an unsatisfied pair $(u, s)$ with smallest $d\left(s_{u}, s\right)$ among all unsastisfied pairs. Let $j$ be the smallest integer such that $d\left(s_{u}, s\right) \leq 2^{j} r$ holds. As in the construction of the proof of Proposition 7 , user $u$ can then receive all subjects in $B_{u, i}=B\left(s_{u}, \min \left(R_{u}, 2^{i} r\right)\right)$ through connections to the nodes in some set $N_{u, i}$ as follows. The set $N_{u, 1}$ contains at most $\gamma \delta$ producers: those within distance $2 r$ from $s_{u}$. For $2 \leq i \leq j$, the set $N_{u, i}$ contains at most $\gamma^{2}$ users such that $B_{u, i}$ is included in $\cup_{v \in N_{u, i}} B_{v, i-1}$. Following these users allows $u$ to receive all subjects in the ring $B_{u, i} \backslash B_{u, i-1}$. The reason is twofold. First, the choice of $(u, s)$ ensures that every user $v$ receives all subjects in $B_{v, i-1}$ as this ball has radius $2^{i-1} r$ at most and $2^{i-1} r \leq$ $2^{j-1} r<d\left(s_{u}, s\right)$ by the choice of $j$. Second, any subject $s \in B_{v, i-1} \backslash B_{u, i-1}$ where $v$ is a user in $N_{u, i}$ is received by $u$ according to expertise-filtering since $d\left(s_{v}, s\right) \leq 2^{i-1} r$ and $d\left(s_{u}, s\right)>2^{i-1} r$. Overall, $u$ can receive all subjects within distance $\min \left(R_{u}, 2^{j} r\right)$ including $s$. Nearest-subject priority implies that the configuration is unstable as long as $\Delta_{u} \geq \gamma \delta+\gamma^{2}(j-1)$ which is the case for $\Delta_{u} \geq \gamma \delta+\gamma^{2} \log \frac{R_{u}}{r}$ since $R_{u} \geq d\left(s_{u}, s\right)>2^{j-1} r$. Since the system must stabilize to some equilibrium according to Proposition 8 , every user $u$ must receive all news about subjects in $S_{u}$ in that stable configuration.

Convergence speed: Let $P_{j}$ denote the property that every user $u$ receives all subjects in his ball of radius $\min \left(R_{u}, 2^{j} r\right)$. We show by induction on $j$ that $P_{j}$ is satisfied after the first sequence of best response moves constituting $j$ rounds. Consider the first moves. The nearest-subject priority 
rule ensures that each user $u$ receives all subjects in $B_{u, 1}$ after his first move. The reason is simply that his budget is sufficient to connect directly to all producers in $B_{u, 1}$ (recall that this ball has size at most $\gamma \delta$ as shown in the proof of Proposition (7). A move later on by a user $v$ cannot alterate the reception of a subject $s$ with $d\left(s_{u}, s\right) \leq 2 r$. This is an effect of the expertise filtering rule: $u$ can be affected only when he receives $s$ by a path from $v$ to $u$ with $d\left(s_{v}, s\right) \leq d\left(s_{u}, s\right) \leq 2 r$ according to expertise filtering and we know that a best response move of $v$ ensures that $v$ will receive all subjects in $B\left(s_{v}, 2 r\right)$ after the move. Property $P_{1}$ is thus satisfied as soon as every user has made a move under best response, that is after the first round. Now assume that $P_{j-1}$ is satisfied. A move by user $u$ cannot incur the loss of a subject $s$ for a user $w$ whose central subject $s_{w}$ is at distance at most $2^{j} r$ from $s$. The reason is that if $w$ receives this subject through a path from $u$, the expertise-filtering rule implies that $s_{u}$ is at distance at most $2^{j} r$ from $s$ also. On the other hand, $P_{j-1}$ implies the necessary conditions to apply the same argument as in the first part of the proof. We can thus show that some move by user $u$ will allow him to receive all subjects within distance $\min \left(R_{u}, 2^{j} r\right)$. As $u$ forwards this subject before the move, we have $d\left(s_{u}, s\right) \leq \min \left(R_{u}, 2^{j} r\right)$ and $u$ still forwards the subject after a best response move. We can thus conclude that if a user $w$ receives all subject within distance $2^{j} r$, he will continue to receive all of them along the $j$ th round. This implies in particular that property $P_{j-1}$ thus remains satisfied along the round. Additionally, a user $u$ receives all subjects in his ball of radius $\min \left(R_{u}, 2^{j} r\right)$ after his first move in the $j$ th round and this is preserved during the sequel of the round. We can thus conclude that $P_{j}$ is satisfied as soon as the $j$ th round is completed and remains satisfied afterwards. This completes the proof by induction. For

$j=\left\lceil\log \frac{R_{m}}{r}\right\rceil, P_{j}$ imply that every user receives all subjects in his interest ball. The convergence time is thus at most $\left\lceil\log \frac{R_{m}}{r}\right\rceil$ rounds.

\section{Budget of attention and cost of connections}

As a simplifying assumption, we have considered up to now that filtering each connection had the same cost. We now discuss how our work can be extended to reflect the fact that the connection $(v, u)$ from a user $u$ to a user $v$ depend on how the interests of $u$ and $v$ differ. A simple idea would be to let the cost be an increasing function of the number of uninteresting 
messages $v$ sends to $u$. However, this would make the model much more complex as costs would then depend on the dynamics. Moreover this would not reflect the reality where a link is usually established on a long term basis. We thus propose to better model the cost of attention of a connection as an increasing function of the number of uninteresting subjects $v$ may potentially bring to $u$, that is $\left|S_{v} \backslash S_{u}\right|$. If we normalize the cost of connecting directly to a producer to 1 , a simple cost function for establishing link $(v, u)$ could be $c(v, u)=1+\alpha_{u}\left|S_{v} \backslash S_{u}\right|$ where $\alpha_{u}>0$ is some parameter comparing the cost of filtering an uninteresting subject for user $u$ to the cost of initiating a connection.

The model remains the same in the homogeneous case. In the heterogeneous case, the negative results of Section 4 remain valid in this more complex model as we could expect. The example with high price of anarchy given in Figure 4(a) can be modified so that the cost of connecting to any user is the same and the bad equilibrium configuration remains stable (it suffices to add $(n / 2-1)-(2 \Delta-3)$ subjects for each pair $a_{i}, b_{i}$ of users that interest both of them and no one else). In the non-convergence example of Figure 5, the two users that oscillate between two strategies are basically interested in the same subjects and they oscillate between users bringing only interesting subjects. The possibility of non-convergence thus remains valid also.

Pushing forward the idea, we can assume that a user tends to accept a certain fraction of uninteresting content compared to interesting content. This could be modelled by setting $\alpha_{u}=\frac{\beta}{\left|S_{u}\right|}$ for some constant $\beta>0$. Additionally, there is no reason for counting several times a subject that is brought by several connections (micro-blogging systems can automatically eliminate duplicates). We can thus estimate globally the cost of the set of connections $F_{u}$ made by user $u$ as:

$$
c\left(F_{u}\right)=\left|F_{u}\right|+\beta \frac{\left|\cup_{(v, u) \in F_{u}} S_{v} \backslash S_{u}\right|}{\left|S_{u}\right|}
$$

Our model with structured interest sets naturally fits with this kind of cost if we make a slightly stricter assumption on the metric, namely that it has bounded growth. Given a constant $\gamma^{\prime}>1$, a metric is $\gamma^{\prime}$-growthbounded if for any point $s$ and radius $R$, the ball $B(s, 2 R)$ is larger than $B(s, R)$ by a multiplicative factor of $\gamma^{\prime}$ at most. This is indeed a special case of doubling metric and still generalizes Euclidean metrics. The expertisefiltering rule implies that a user $u$ follows users at distance at most $2 R_{u}$. 
We can adapt the interest-radius smoothness assumption by requiring that for any users $u, v$ with $d\left(s_{u}, s_{v}\right) \leq 4 \max \left(R_{u}, R_{v}\right)$, we have $R_{v} \geq R_{u} / 2$ and $R_{u} \geq R_{v} / 2$. This setting thus implies $\cup_{(v, u) \in F_{u}} S_{v} \subseteq B\left(u, 4 R_{u}\right)$, and the size of this ball is at most $\gamma^{\prime 2}$ times larger than $B\left(u, R_{u}\right)=\left|S_{u}\right|$ by the $\gamma^{\prime}$-growthbounded hypothesis. We thus get $c\left(F_{u}\right) \leq\left|F_{u}\right|+\beta \gamma^{\prime 2}$. Expertise-filtering and smoothness assumptions on the metric modeling the interests thus imply that the cost term for the filtering of uninteresting content is upper-bounded by a constant. The results presented in Section 5 thus still apply up to the corresponding additive term in the budget of attention bounds. We thus see that this finer model gives another justification to expertise-filtering. This may indeed reveal that the cost of filtering may naturally induce an incentive for applying expertise-filtering.

\section{Concluding remarks}

We have shown that a flow game can have complex dynamics that may not converge. However, we can prove convergence to efficient equilibrium for both homogeneous flow games (with very weak assumptions) and metric flow games (with more technical assumptions). While our proofs give exponential bounds on convergence time in general, we get linear convergence time up to a logarithmic factor (in number of moves) for structured interest set with expertise-filtering and nearest-subject priority, showing that understanding the structure of interests and its relation to forwarding mechanisms is a key aspect of information flow in social networks. Direct follow up of this work concerns the study of the speed of convergence in general and the characterization of flow games having pure Nash equilibria.

A dual variant of our model could be to consider that every user gathers all the subjects he is interested in while he tries to minimize the required cost of attention. We could also mix both models, using utility functions combining coverage of interest set and cost of attention (the function being increasing in the number of interesting subjects received and decreasing in the costs of attention of the formed links). Another interesting variant resides in considering the size of flows or equivalently their rate of news. The budget of attention required to follow a flow should then increase accordingly to its size. This variant is complementary to weighting flows as a flow with more news might be weighted higher by users wishing to follow it.

In that context, we believe the two following directions are promising for 
efficient social dissemination. First, incentive mechanisms, e.g. reputation counters maintained by users, or payments between users, may be a complementary approach to augment the performance of self-organizing social flows. Second, more elaborate content filtering between contact-follower pairs may also lead to substantial improvements. We have already introduced expertise filtering, which could translate into implementable mechanisms in existing social networking platforms. More generally there appears to be a rich design space of filtering rules based on combinations of interests and expertise.

\section{References}

[1] I. Abraham, D. Malkhi, and O. Dobzinski. LAND: stretch $(1+\epsilon)$ localityaware networks for DHTs. In J. I. Munro, editor, SODA, pages 550-559. SIAM, 2004.

[2] E. Anshelevich, A. Dasgupta, J. Kleinberg, E. Tardos, T. Wexler, and T. Roughgarden. The price of stability for network design with fair cost allocation. In Proceedings of the 45th Annual IEEE Symposium on Foundations of Computer Science, FOCS '04, pages 295-304, Washington, DC, USA, 2004. IEEE Computer Society.

[3] V. Bala and S. Goyal. A noncooperative model of network formation. Econometrica, 68(5):1181-1229, 2000.

[4] X. Bei, W. Chen, S.-H. Teng, J. Zhang, and J. Zhu. Bounded budget betweenness centrality game for strategic network formations. Theor. Comput. Sci., 412(52):7147-7168, Dec. 2011.

[5] D. Black. The theory of committees and elections. Springer, 1958.

[6] M. Cha, H. Haddadi, F. Benevenuto, and P. K. Gummadi. Measuring user influence in twitter: The million follower fallacy. In W. W. Cohen and S. Gosling, editors, ICWSM. The AAAI Press, 2010.

[7] K. Clarkson. Nearest neighbor queries in metric spaces. Discrete \&6 Computational Geometry, 22(1):63-93, 1999.

[8] A. Fabrikant, A. Luthra, E. Maneva, C. H. Papadimitriou, and S. Shenker. On a network creation game. In Proc. ACM PODC, pages 347-351, 2003. 
[9] A. Fabrikant, C. H. Papadimitriou, and K. Talwar. The complexity of pure nash equilibria. In L. Babai, editor, STOC, pages 604-612. ACM, 2004.

[10] P. Fraigniaud, E. Lebhar, and L. Viennot. The inframetric model for the internet. In Proceedings of the 27th IEEE International Conference on Computer Communications (INFOCOM), pages 1085-1093, Phoenix, 2008.

[11] A.-T. Gai, D. Lebedev, F. Mathieu, F. De Montgolfier, J. Reynier, and L. Viennot. Acyclic Preference Systems in P2P Networks. In Proc. Euro-Par, 2007.

[12] G. Giakkoupis. Tight bounds for rumor spreading with vertex expansion. In Proceedings of the Twenty-Fifth Annual ACM-SIAM Symposium on Discrete Algorithms, SODA 2014, Portland, Oregon, USA, January 5-7, 2014, pages 801-815, 2014.

[13] S. Har-Peled and M. Mendel. Fast construction of nets in low dimensional metrics, and their applications. In J. S. B. Mitchell and G. Rote, editors, Symposium on Computational Geometry, pages 150-158. ACM, 2005 .

[14] T. H. Haveliwala. Topic-sensitive pagerank: A context-sensitive ranking algorithm for web search. IEEE Trans. Knowl. Data Eng., 15(4):784796, 2003.

[15] M. Jackson. Social and Economic Networks. Princeton University Press. Princeton University Press, 2010.

[16] B. Jiang, N. Hegde, L. Massoulié, and D. Towsley. How to optimally allocate your budget of attention in social networks. In Proc. IEEE Infocom, 2013.

[17] D. R. Karger and M. Ruhl. Finding nearest neighbors in growthrestricted metrics. In J. H. Reif, editor, STOC, pages 741-750. ACM, 2002.

[18] D. Kempe, J. M. Kleinberg, and É. Tardos. Maximizing the spread of influence through a social network. In Proceedings of the Ninth ACM 
SIGKDD International Conference on Knowledge Discovery and Data Mining, pages 137-146, 2003.

[19] N. Laoutaris, L. J. Poplawski, R. Rajaraman, R. Sundaram, and S.H. Teng. Bounded budget connection (BBC) games or how to make friends and influence people, on a budget. In Proceedings of the twentyseventh ACM symposium on Principles of distributed computing, PODC '08, pages 165-174, New York, NY, USA, 2008. ACM.

[20] L. Massoulié and A. Twigg. Rate-optimal schemes for peer-to-peer live streaming. Perform. Eval., 65(11-12):804-822, 2008.

[21] A. May, A. Chaintreau, N. Korula, and S. Lattanzi. Filter \& follow: How social media foster content curation. In The 2014 ACM International Conference on Measurement and Modeling of Computer Systems, SIGMETRICS '14, pages 43-55, New York, NY, USA, 2014. ACM.

[22] D. Monderer and L. Shapley. Potential games. Games and Economic Behavior, pages 124-143, 1996.

[23] N. Nisan, T. Roughgarden, É. Tardos, and V. V. Vazirani, editors. Algorithmic Game Theory. Cambridge Univ Press, 2007.

[24] C. G. Plaxton, R. Rajaraman, and A. W. Richa. Accessing nearby copies of replicated objects in a distributed environment. Theory Comput. Syst., 32(3):241-280, 1999.

[25] R. W. Rosenthal. A class of games possessing pure-strategy Nash equilibria. International Journal of Game Theory, 2:65-67, 1973.

[26] T. Roughgarden. Potential functions and the inefficiency of equilibria. Proceedings of the International Congress of Mathematicians (ICM), 3:1071-1094, 2006.

[27] H. A. Simon. Designing organizations for an information rich world. In M. Greenberger, editor, Computers, communications, and the public interest, pages 37-72. The Johns Hopkins Press, Baltimore, 1971. 